\title{
Estudo EVAPE (Exploring Vascular Protective Effects)
}

Idalmiro Carraça,* Prado Amorim, * Guilherme Ferreira, * Celeste Legrant,* Matilde Pinto*

\section{RESUMO}

Objectivos: Avaliar retrospectiva e comparativamente o efeito de três classes terapêuticas na mortalidade de causa vascular, em utentes dos cuidados de saúde primários sujeitos aos padrões habituais de prescrição médica; avaliar o contributo relativo dos factores de risco para a morte de causa vascular.

Tipo de estudo: Estudo de casos e controlos.

Local: Centro de Saúde dos Olivais

População: Utentes dos cuidados de saúde primários: casos (149): óbitos por causa vascular; controlos (253): seleccionados aleatoriamente de entre os utentes que frequentaram regularmente as consultas dos respectivos médicos de 2002 a 2005.

Métodos: A metodologia consistiu, na prática, em recuar no tempo e avaliar numa dada população, que diferenças se registavam, face a um grupo de controlo, em termos terapêuticos e de factores de risco, que possam estar associados ao desfecho (morte de causa vascular).

Testes Estatísticos: Qui-quadrado para testar homogeneidade das amostras nas variáveis, sexo, idade, factores de risco e terapêutica; cálculo do Odds Ratio (OR), como estimativa do risco de morte de causa vascular por classe terapêutica. Regressão logística (OR ajustado aos factores de risco); cálculo do OR no estudo de determinadas associações terapêuticas. Intervalo de confiança de $95 \%$.

Resultados: O grupo terapêutico das estatinas esteve associado a uma redução estimada do risco de morte de causa vascular (65\% a 81\%). Esta associação manteve-se independentemente dos factores de risco (OR ajustado para os mesmos na regressão logística);

Conclusões: O grupo terapêutico das estatinas revelou associação significativa com a redução do risco de morte de causa vascular, independentemente de estar associada ou não a outras terapêuticas. De entre os factores de risco que mais contribuíram para a mortalidade cv destacaram-se o tabagismo e os eventos cv prévios.

Palavras-Chave: Risco Cardiovascular; Terapêutica Preventiva.

\section{INTRODUÇÃO}

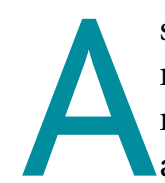

s situações de risco cardiovascular (cv) são responsáveis por importante morbilidade $\mathrm{e}$ mortalidade, destacando-se como causa mais associada à morte prematura - anualmente 4 milhões de mortes na Europa, ou $49 \%$ de todas as mortes, das quais $30 \%$ em indivíduos abaixo dos 65 anos. ${ }^{1}$ Inúmeros ensaios clínicos aleatórios e controlados evidenciam o efeito protector de diversas terapêuticas em pacientes com risco cardiovascular: antiagregantes plaquetários, estatinas e os novos antihipertensores (iecas/aras II) revelaram efectiva redução do risco cv. Estes efeitos revelam-se após terapêuticas continuadas por períodos de tempo variáveis, por vezes de poucos

*Médico de Medicina Geral e Familiar do Centro de Saúde dos Olivais meses, em situações de alto risco, ou ao fim de alguns anos, na prevenção primária. Os inibidores da enzima de conversão (iecas)/antagonistas dos receptores da angiotensina (aras II) reduzem a morbi-mortalidade cv nos pacientes com variadas situações, ${ }^{2}$ nomeadamente, hipertensão (sistólica), ${ }^{3-5}$ disfunção assintomática do ventrículo esquerdo, ${ }^{6}$ insuficiência cardíaca manifesta, quer após enfarte do miocárdio, ${ }^{7-10}$ quer em situação crónica, ${ }^{11,12}$ doença coronária estável. ${ }^{13}$ As estatinas reduzem a morbi-mortalidade coronária e cérebro vascular em pacientes com: hipertensão, com ou sem dislipidémia, ${ }^{14}$ doença coronária, ${ }^{15-21}$ síndromes coronários agudos ou instáveis, ${ }^{22-24}$ doença arterial periférica. ${ }^{20}$

Os antiagregantes plaquetários reduzem a morbi-mortalidade cv em pacientes com diferentes situações de risco, em que se demonstra uma redução global do 
risco cv, não obstante discrepâncias entre a redução do risco de enfarte do miocárdio e do risco de acidente vascular cerebral (AVC)..$^{25-34}$

Da revisão dos grandes ensaios clínicos controlados, atrás citados, marcos da investigação e da medicina baseada na evidência, destacamos os seguintes, por grupo terapêutico: Iecas/aras II: LIFE 2002, SOLVD 1992, ${ }^{6}$ SAVE 1993, ${ }^{7}$ AIRE $1993,{ }^{8}$ TRACE $1995,{ }^{9}$ HOPE 2000, ${ }^{10}$ CHARM 2003, ${ }^{12}$ EUROPA 2003, ${ }^{13}$ Estatinas: $4 S$ 1994, ${ }^{15}$ WOSCOOPS 1995, ${ }^{16}$ CARE 1998, ${ }^{17}$ LIPS 2002, ${ }^{18}$ HPS 2002, ${ }^{20}$ AFSCAPS / Tex CAPS 1998, ${ }^{2}$ LIPID 1998, ${ }^{23}$ MIRACLE 2001, ${ }^{24}$ SCAT 2000, ${ }^{35}$ ALLHAT 2002. ${ }^{36}$ Antiagregantes plaquetários: ATC 1988, ${ }^{25}$ CAPRIE 1996, ${ }^{30}$ ISIS-2 1988, ${ }^{31}$ CURE 2001. ${ }^{33}$ Antidiabéticos: STENO 2 2003. ${ }^{37}$

A demonstração da efectividade dos tratamentos preventivos tem-se acompanhado duma evolução no sentido de deslocar as situações elegíveis, de risco elevado para risco moderado, preconizando-se a antecipação progressiva do início dos tratamentos, nomeadamente a nível da prevenção primária. Esta tendência verifica-se, quer para os anti-hipertensores, quer para as estatinas, como se pode constatar na evolução das guidelines, nos últimos anos, reiteradamente propondo metas cada vez mais agressivas e ambiciosas e tratamentos mais precoces. ${ }^{1} \mathrm{Na}$ verdade, o prognóstico de curto prazo, em situações específicas de elevado risco cv, tem vindo a ser alterado em função dos resultados obtidos nos grandes ensaios clínicos, continuando a debater-se a importância e a contribuição relativa para esses resultados, por um lado, dos factores de risco cv prévios (isoladamente ou em associação) e, por outro, da terapêutica agressiva. Independentemente dos níveis de colesterol iniciais, ou de outros factores de risco, alguns estudos revelaram que a terapêutica agressiva pode mudar o prognóstico das doenças cardiovasculares. ${ }^{20,21,24,37} \mathrm{O}$ mesmo racional suporta a importância do controlo agressivo da tensão arterial ou da diabetes.

Tendo em conta a evidência científica acumulada, pode-se questionar a ausência de estudos que testem no terreno da clínica os efeitos de protecção cv associados àquelas terapêuticas. Estudos de casos e controlos (com rigorosa selecção aleatória), em populações dos cuidados de saúde primários, mediante avaliação da morbilidade e da mortalidade (de causa vascular, por eventos específicos ou por todas as causas), dos factores de risco e dos tratamentos em curso, permitem pes- quisar, numa óptica retrospectiva, diferenças no risco estimado de morte de causa vascular associadas aquelas terapêuticas. É este o principal objectivo do presente trabalho, admitindo como hipótese a de verificar no terreno da clínica que qualquer dos grupos terapêuticos considerados contribui para a redução do risco de morte de causa vascular. Uma segunda hipótese admite a existência de resultados positivos nos doentes medicados com associações daqueles grupos terapêuticos, a explorar parcial e comparativamente. Pretende-se também explorar, neste estudo, o contributo relativo dos factores de risco para a morte de causa vascular.

\section{MÉTODOS}

Tipo de estudo: casos e controlos.

Desenho: a metodologia consistiu, na prática, em recuar no tempo e avaliar, numa dada população, que diferenças se registavam, face a um grupo de controlo, em termos terapêuticos e de factores de risco, que possam estar associadas ao desfecho - morte de causa vascular.

Local e tempo de duração: estudo realizado de 2006 a 2007, com recolha de dados que abrangeu retrospectivamente os anos de 2002 a 2006.

População-alvo: utentes dos cuidados de saúde primários com 50 ou mais anos de idade, dos dois sexos.

Métodos de amostragem: os casos foram identificados a partir da base de dados de óbitos do centro de saúde, tendo-se seleccionado a totalidade dos doentes cuja morte ocorreu nos anos de 2003 ou 2004, por causa vascular no registo de óbito. ${ }^{39}$ Os controlos foram seleccionados aleatoriamente (aleatorização sistemática), de entre os 6.219 utilizadores regulares das consultas dos respectivos médicos de família - mínimo de 2 consultas em cada ano (2002, 2003, 2004 e 2005), previamente identificados, respeitando quotas por sexo e escalão etário para assegurar a homogeneidade das amostras. Considerou-se suficiente para os objectivos do estudo um número de controlos de cerca do dobro dos casos.

Unidades de observação: utentes incluídos no estudo.

Variáveis medidas: sexo e idade (ano de nascimento); factores de risco: hipertensão, doença coronária, disfunção cardíaca, doença arterial periférica, fibrilhação auricular, eventos cv, diabetes, tabagismo, obesidade, dislipidémia; associação de três ou mais dos anteriores factores de risco; terapêuticas prescritas: iecas/aras II, antiagregantes plaquetários (asp), estatinas, 
diuréticos, «outros anti-hipertensores», antagonistas do cálcio, anticoagulantes; óbito por causa vascular $(0,1)$, que corresponde ao endpoint.

Métodos de recolha de dados e instrumentos utilizados: construção prévia de grelha para colheita de dados proveniente de certidão de óbito, de registos no processo clínico, complementada com entrevista semi-estruturada aos respectivos médicos assistentes, registo de contactos SINUS.

Critérios: critérios de inclusão: utentes do centro de saúde dos Olivais, de ambos os sexos, com 50 ou mais anos de idade, com prescrição médica (usual) por períodos de 1 ou mais anos de terapêutica continuada; confrontada a dose prescrita com a dose diária recomendada (tabela empírica com intervalo definido, segundo o prontuário terapêutico, ${ }^{38}$ considerando-se consignadas as terapêuticas dentro do respectivo limiar terapêutico; critérios de exclusão: utentes com informação ambígua/insuficiente, ou fora dos critérios de inclusão; critério clínico na definição/medição das variáveis factores de risco, terapêuticas prescritas e óbito de causa vascular (conjugação com dados do processo clínico e entrevista semi-estruturada com o médico assistente); admitido efeito de classe dos três grupos terapêuticos considerados, não obstante as diferenças existentes; reunidos num mesmo grupo fármacos com acções similares, diuréticos, antagonistas do cálcio, beta-bloqueantes, anticoagulantes, sob as respectivas designações; miscelânea de fármacos anti-hipertensores heterogéneos reunidos sob a designação de «outros anti-hipertensores».

Plano de análise estatística: qui quadrado para testar homogeneidade das amostras nas variáveis, sexo, factores de risco e terapêutica ( $t$-test para a idade). Tratando-se de estudo exploratório a análise procedeu nos seguintes passos: 1. Cálculo do Odds Ratio (OR), como estimativa do risco de morte de causa vascular por factores de risco e por classe terapêutica. 2. Controlo de variáveis confundentes, isto é, que estejam duplamente associadas (endpoint e terapêutica) - análise em subgrupos de risco e recurso à regressão logística $(O R$ ajustado aos factores de risco duplamente associados ao endpoint e à terapêutica). Tratando-se dum estudo de casos e controlos as estimativas de variação do risco relativo $(R R)$ serão efectuadas por aproximação do $O R$ ao $R R$ ). Procedeu-se à agregação de categorias das variáveis para cálculo do $O R$ em tabelas de $2 \times 2$ e à construção de novas variáveis para estudo de associações terapêuticas, duplas e triplas, e para estudo de benefícios adicionais duma determinada classe terapêutica. Foi considerado o intervalo de confiança de $95 \%$.

O tratamento estatístico foi realizado com agregação de dados codificados, salvaguardando o anonimato dos utentes incluídos.

\section{RESULTADOS}

Os resultados resumem-se no texto, quadros e figuras seguintes:

\section{Características dos Pacientes, Factores de Risco e Grupos Terapêuticos}

O estudo englobou 402 doentes, 149 casos e 253 controlos. A média de idades foi sensivelmente igual nos dois grupos (77 anos), com ligeiro predomínio de mulheres em cada grupo.

As amostras não apresentam diferenças significativas na distribuição por sexo e idade, mas divergem significativamente na maioria dos factores de risco: hipertensão, doença coronária, disfunção cardíaca, doença arterial periférica, fibrilhação auricular, eventos cv, diabetes e tabagismo - mais frequentes nos casos. As amostras são semelhantes para obesidade. A dislipidémia predomina significativamente nos controlos (Quadro I).

Casos e controlos divergem significativamente na prescrição das seguintes terapêuticas: diuréticos, estatinas, «outros anti-hipertensores»e anticoagulantes (Quadro II).

A maioria dos doentes do controlo estavam medicados com iecas/aras II, antiagregantes, diuréticos e estatinas, enquanto nos casos predominavam terapêuticas com diuréticos, iecas/aras II, antiagregantes plaquetários e antagonistas do cálcio (Quadro II).

Os iecas/aras II, os antagonistas do cálcio, os beta-bloqueantes e os antiagregantes plaquetários distribuem-se de forma similar nos casos e nos controlos.

Estimativa da redução do risco por grupo terapêutico Os resultados revelam que as estatinas estão associadas, nos pressupostos deste estudo, a uma redução estimada do risco relativo de morte de causa vascular de $65 \%$ (OR: 0,346 IC: 0,215 - 0,558) (Quadro II, Figura 1, aproximação ao $R R$ pelo $O R$ ). 


\begin{tabular}{|c|c|c|c|c|c|c|c|}
\hline \multirow[b]{2}{*}{ Variáveis } & \multicolumn{2}{|c|}{ Controlos } & \multicolumn{2}{|c|}{ Casos } & \multirow[b]{2}{*}{ OR } & \multirow[b]{2}{*}{ IC 95\% } & \multirow[b]{2}{*}{$\mathbf{P}$} \\
\hline & $\mathbf{n}$ & $\%$ & $\mathrm{n}$ & $\%$ & & & \\
\hline \multicolumn{8}{|l|}{ Sexo } \\
\hline Masculino & 123 & 48,6 & 72 & 48,3 & \multirow{3}{*}{1,012} & \multirow{3}{*}{$0,675-1,517$} & \multirow{3}{*}{0,955} \\
\hline Feminino & 130 & 51,4 & 77 & 51,7 & & & \\
\hline & 253 & 100 & 149 & 100 & & & \\
\hline \multirow{2}{*}{$\begin{array}{l}\text { Idade (média) } \\
\text { (SD) }\end{array}$} & \multicolumn{2}{|r|}{76,91} & \multicolumn{2}{|r|}{77,42} & & & \multirow[t]{2}{*}{0,592} \\
\hline & \multicolumn{2}{|r|}{9,217} & \multicolumn{2}{|r|}{9,181} & & & \\
\hline Hipertensão Arterial & 187 & 74 & 122 & 82,4 & 1,656 & $1,001-2,752$ & 0,050 \\
\hline D Coronária & 62 & 24,5 & 67 & 45,3 & 2,548 & $1,654-3,926$ & $<0,001$ \\
\hline Disfunção Cardíaca & 45 & 17,8 & 56 & 37,8 & 2,814 & $1,771-4,469$ & $<0,001$ \\
\hline Fib Auricular & 23 & 9,1 & 32 & 21,6 & 2,759 & $1,544-4,929$ & $<0,001$ \\
\hline Eventos cV & 37 & 14,6 & 54 & 36,7 & 3,390 & $2,090-5,499$ & $<0,001$ \\
\hline Diabetes & 54 & 21,3 & 55 & 37,2 & 2,179 & $1,391-3,415$ & 0,001 \\
\hline Obesidade & 65 & 26 & 37 & 26,8 & 1,043 & $0,651-1,670$ & 0,862 \\
\hline Tabagismo & 16 & 6,5 & 28 & 21,4 & 3,925 & $2,035-7,568$ & $<0,001$ \\
\hline D Art Periférica & 21 & 6,5 & 31 & 20,9 & 2,915 & $1,604-5,294$ & $<0,001$ \\
\hline Dislipidémia & 131 & 52 & 58 & 39,2 & 0,595 & $0,394-0,899$ & 0,013 \\
\hline 3 ou + F Risco & 116 & 46,2 & 103 & 70,5 & 2,788 & $1,806-4,302$ & $<0,001$ \\
\hline
\end{tabular}

Obs. média de idade: 77,1 mediana: 78,0; SD: 9,196; mínima: 51; máxima: 98; OR: Odds Ratio; Qui quadrado: P: 0,05; Cl: $95 \%$.

QUADRO II. Resultados por Grupo Terapêutico: Odds Ratio (OR)
\begin{tabular}{|l|c|c|c|c|c|c|c|}
\hline \multirow{2}{*}{ Variáveis } & \multicolumn{2}{c|}{ Controlos } & \multicolumn{2}{c|}{ Casos } & \\
\cline { 2 - 9 } & $\mathbf{n}$ & $\%$ & $\mathbf{n}$ & $\%$ & OR & IC $95 \%$ & P \\
\hline lecas/Aras II & 152 & 60,1 & 86 & 57,7 & 0,907 & $0,601-1,368$ & 0,642 \\
\hline Diuréticos & 113 & 44,7 & 86 & 58,1 & 1,719 & $1,141-2,589$ & 0,009 \\
\hline Antag cálcio & 63 & 24,9 & 47 & 32 & 1,417 & $0,905-2,220$ & 0,127 \\
\hline Beta bloqueantes & 42 & 16,6 & 18 & 12,1 & 0,690 & $0,381-1,250$ & 0,219 \\
\hline Estatinas & 105 & 41,5 & 29 & 19,7 & 0,346 & $0,215-0,558$ & $<0,001$ \\
\hline Antiagregantes & 124 & 49,2 & 64 & 43 & 0,777 & $0,517-1,168$ & 0,225 \\
\hline Outros anti-hipertensores & 18 & 7,1 & 25 & 16,9 & 2,654 & $1,394-5,052$ & 0,002 \\
\hline Anticoagulantes & 6 & 2,4 & 13 & 8,7 & 3,935 & $1,463-10,587$ & 0,004 \\
\hline
\end{tabular}

Obs. OR: Odds Ratio; Qui quadrado P: 0,05; Cl: $95 \%$.

Pelo contrário, os resultados associam os diuréticos a pior prognóstico (OR: 1,719 IC: 1,141-2,589).

Os restantes grupos terapêuticos não apresentaram diferenças significativas para o endpoint.

\section{Controlo de variáveis de confundimento}

O estudo em subgrupos de risco e a regressão logística ajustada aos factores de risco mostrou os seguintes re- sultados (Quadros III, IV e V)

O Quadro VI sintetiza a estimativa da variação do risco por grupo terapêutico.

\section{Associações Terapêuticas}

As estatinas, quando associadas a outros fármacos, mantiveram a associação com menor mortalidade (Quadro VII). Destacam-se os resultados com a associa- 


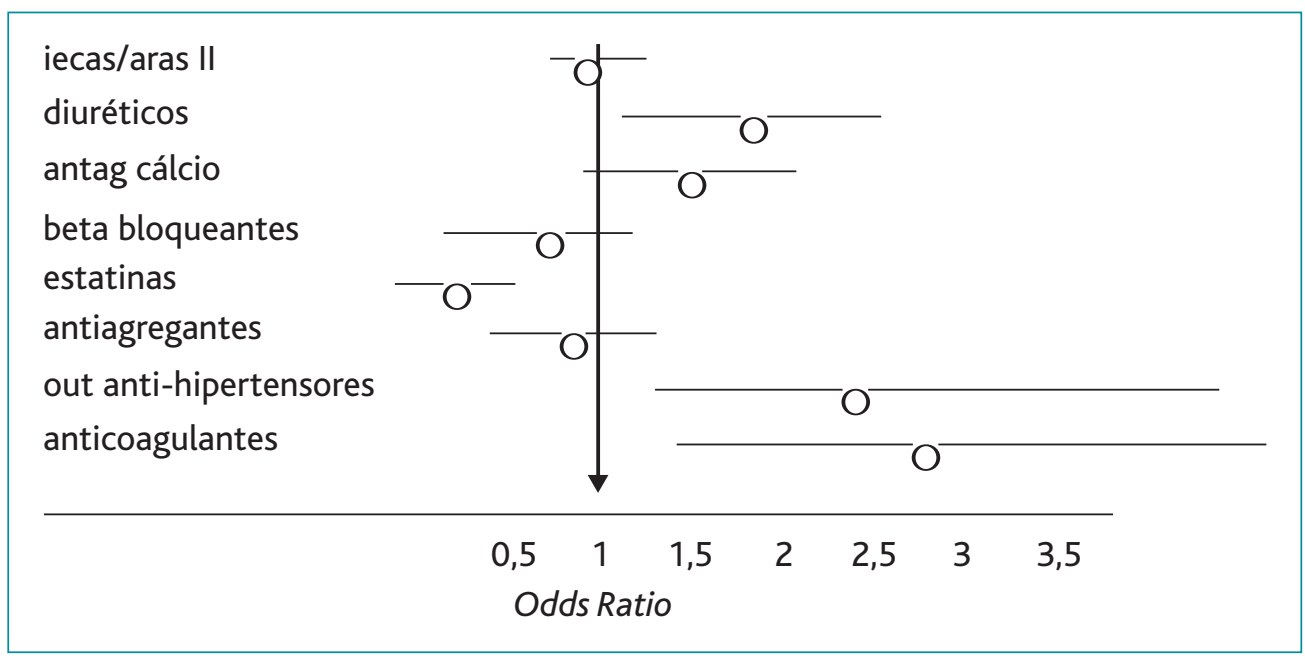

Figura 1. Estimativa da redução RR por grupo terapêutico (OR).

ção de estatinas e antiagregantes, e também com a associação de estatinas e iecas/aras II.

Nos doentes sujeitos a estatinas, a associação com antiagregantes (ou com iecas/aras II), não revelou qualquer benefício adicional destes fármacos. Pelo contrário, considerando os doentes sob antiagregantes, divididos em dois grupos consoante faziam estatinas ou não, os resultados revelam diferenças significativas no sentido do benefício adicional da inclusão de estatinas.

O mesmo benefício adicional das estatinas se verificou nos doentes medicados com iecas/aras II.

\section{Contributo dos factores de risco para o endpoint}

Os resultados sugerem que o risco relativo de morte de causa vascular foi substancialmente agravado pela presença de tabagismo e pela existência de eventos cv prévios, à cabeça, e ainda pela presença de qualquer uma das situações seguintes: doença arterial periférica, disfunção cardíaca, três ou mais factores de risco, fibrilhação auricular, doença coronária ou diabetes.

A dislipidémia, de acordo com os resultados deste estudo, não parece ter concorrido para o desfecho, estando inclusive associada a uma redução do risco relativo.

\section{DISCUSSÃO DOS RESULTADOS}

Nos pressupostos deste estudo, eram esperadas associações de cada uma das classes terapêuticas principais com menor mortalidade, o que só se verificou com as estatinas. Os resultados dos diuréticos também sobres- saíram mas pela negativa. Estatinas e diuréticos revelaram resultados diferentes na redução do risco relativo para o endpoint (aproximação pelo $O R$ ). Estes achados, em parte inesperados e surpreendentes, obrigaram a considerar eventuais enviesamentos na selecção das amostras. De facto, casos e controlos diferem em factores de risco e nalgumas terapêuticas e estas diferenças influenciam os resultados. A dupla associação entre alguns factores de risco e terapêutica com a mortalidade coloca a hipótese de confundimento. Até que ponto a maior prevalência de dislipidémia nos controlos, e a maior utilização das estatinas nesse grupo, determinam, por si só, a associação das estatinas a menor mortalidade? - do ponto de vista teórico não é crível que a dislipidémia se associe a menor mortalidade, mas exige-se estratégia para controlar possíveis variáveis confundentes: 1 . restrição populacional, estudando o fenómeno na sub-população definida pela variável confundente, que assim deixa de interferir nos resultados - confrontando casos e controlos para as estatinas na população com dislipidémia; foram ainda analisados outros subgrupos de risco pertinentes, tendo em vista apurar eventuais diferenças nos resultados; estes procedimentos, visando multiplicar os ângulos de visão para melhor interpretar os resultados, derivam da impossibilidade de isolar totalmente os doentes com determinados factores de risco e sob determinada terapêutica, dada a dimensão das amostras, a comorbilidade e as indicações de cada terapêu- 


\begin{tabular}{|c|c|c|c|c|}
\hline Grupo Terapêutico & sub-população & OR & IC & estimativa var. $\mathbf{R R}^{*}$ \\
\hline \multirow{5}{*}{ Estatinas } & evento cv prévio & 0,228 & $0,094-0,556$ & $-77 \%$ \\
\hline & $\geq 3$ factores risco & 0,271 & $0,153-0,4797$ & $-73 \%$ \\
\hline & dislipidémia & 0,279 & $0,142-0,547$ & $-72 \%$ \\
\hline & diabéticos & 0,389 & $0,176-0,861$ & $-61 \%$ \\
\hline & doença coronária & 0,622 & $0,303-1,277$ & NS \\
\hline \multirow{5}{*}{ Diuréticos } & evento cv prévio & 2,053 & $0,875-4,817$ & NS \\
\hline & diabéticos & 1,619 & $0,756-3,468$ & NS \\
\hline & disf. cardíaca & 1,538 & $0,619-3,817$ & NS \\
\hline & hipertensão & 1,418 & $0,789-2,286$ & NS \\
\hline & $\geq 3$ factores risco & 1,818 & $1,036-3,191$ & $+82 \%$ \\
\hline
\end{tabular}

*Estimativa var RR: estimativa de variação do risco relativo (OR-1) x100. $\mathrm{P}<0,05$

QUADRO IV. Regressão logística: estatinas - OR ajustado para os factores de risco
\begin{tabular}{l|c|c|c|c|c|c|c|c|} 
Grupo terapêutico & $\mathbf{0}$ & $\mathbf{1}$ & $\mathbf{P}$ & & & \\
\hline Estatinas & $\mathbf{0}$ & 144 & 0,000 & 0,76 & $69,8 \%$ & 0,190 & $0,092-0,393$ & 0,000 \\
\hline Hipert Arterial & & & & & & 1,067 & $0,581-1,959$ & 0,835 \\
\hline Eventos cv & & & & & & 2,752 & $1,536-4,932$ & 0,001 \\
\hline Dislipidémia & & & & & & 1,084 & $0,555-2,115$ & 0,814 \\
\hline FR>2 & & & & & & 3,004 & $1,667-5,412$ & 0,000 \\
\hline
\end{tabular}

*Valor P do Modelo. **Hosmer and Lemeshow test. ***Teste aos Coeficientes de Regressão

QUADRO V. Regressão logística: diuréticos - OR ajustado para os factores de risco
\begin{tabular}{l|c|c|c|c|c|c|c|c} 
Grupo terapêutico & $\mathbf{0}$ & $\mathbf{1}$ & $\mathbf{P}$ & & \\
\hline Diuréticos & 250 & 145 & 0,000 & 0,491 & $69,4 \%$ & 1,391 & $0,838-2,308$ & 0,201 \\
\hline Hipert Arterial & & & & & & 0,857 & $0,448-1,639$ & 0,641 \\
\hline D. Coronarária & & & & & & 1,245 & $0,729-2,127$ & 0,422 \\
\hline Disf Cardíaca & & & & & & 1,542 & $0,894-2,660$ & 0,120 \\
\hline Eventos cv & & & & & & 2,029 & $1,162-3,542$ & 0,013 \\
\hline Dislipidémia & & & & & & 0,414 & $0,251-0,683$ & 0,001 \\
\hline FR>2 & & & & & & 2,289 & $1,186-4,419$ & 0,014
\end{tabular}

*Valor P do Modelo. ** Hosmer and Lemeshow test. ${ }^{* * *}$ Teste aos Coeficientes de Regressão

tica; 2. regressão logística ajustada aos factores de risco duplamente associados ao endpoint e à terapêutica (para além da dislipidémia). Esse procedimento revelou de modo consistente, tanto na restrição populacional como na regressão logística, o seguinte: as estatinas mantiveram associação significativa com menor mortalidade em pacientes com dislipidémia, eventos cv prévios, diabetes e «três ou mais factores de 
QUADRO VI. Estimativa da Variação do Risco Relativo por Grupo Terapêutico no global

\begin{tabular}{l|c|c} 
Grupo Terapêutico & $\begin{array}{c}\text { Estimativa } \\
\text { Redução RR* } \\
(\text { OR-1) } \times 100\end{array}$ & $\begin{array}{c}\text { OR } \\
\text { ajustado } \\
\text { aos FR }\end{array}$ \\
\hline Estatinas & $-65 \%$ & $-81 \%$ \\
\hline Diuréticos & $+72 \%$ & NS \\
\hline lecas/ARAS I & NS & \\
\hline Antiagregantes & NS & \\
\hline Antagonistas Cálcio & NS & \\
\hline Beta-Bloqueantes & NS & \\
\hline Anticoagulanttes & $+60 \%$ & \\
\hline «Outros antihipertensores» & $+165,4 \%$ & \\
\hline
\end{tabular}

risco associados», mas não nos pacientes com doença coronária; esta excepção tem interesse, pois a dislipidémia é, como se sabe, um dos factores mais relacionados com a doença coronária, sendo que, nesta situação, a diferença percentual de casos e controlos medicados com estatinas era menor ( $32 \%$ e $43 \%)$; no subgrupo de doentes com dislipidémia as estatinas mantiveram-se associadas a menor mortalidade, anulando-se aqui qualquer possibilidade de enviesamento, como atrás dito (Quadro III); finalmente, a regressão logística, ajustando o OR aos factores de risco (duplamente associados à terapêutica e ao endpoint), as estatinas mantiveram associação a menor mortalidade, com controlo das variáveis de confundimento; de realçar que, na regressão logística, a dislipidémia perdeu o carácter protector, que do ponto de vista do conhecimento actual não fazia qualquer sentido; a estimativa da redução do risco relativo de morte cardiovascular foi ainda reforçado de 65 para $81 \%((O R-1) x 100 . P<0,001)$ (Quadro IV).

Os diuréticos, quando estudados «isoladamente» em sub populações de risco com hipertensão, eventos cv prévios, disfunção cardíaca ou diabetes, não revelaram quaisquer diferenças significativas (Quadro III). No entanto, na subpopulação com «três ou mais factores de risco», os diuréticos mantiveram-se associados ao desfecho. O OR, ajustado aos factores de risco, deixou de ser significativo, no global (Quadro V).

O grupo terapêutico dos anticoagulantes apresentou resultados desfavoráveis, como esperado, dadas as si- tuações de maior risco em que é prescrito (OR: 3,935 IC:1,463 - 10,587). Da mesma forma, o grupo «outros antihipertensores» teve também resultados desfavoráveis, mas trata-se de grupo residual (25 casos e 18 controlos) constituído por miscelânea de fármacos (OR: 2,654 IC: 1,394-5,052).

Embora os resultados do OR coloquem os antiagregantes, beta-bloqueantes e iecas/aras II na faixa protectora (OR inferior à unidade), verifica-se grande dispersão do intervalo de confiança (maior para os iecas/ laras II que para beta-bloqueantes e antiagregantes).

Pode afirmar-se que os casos estavam sujeitos, tendencialmente, a medicações mais «antigas» tais como miscelânea de «outros anti-hipertensores», diuréticos e antagonistas do cálcio e esta circunstância teve importância para os resultados obtidos. O período de terapêutica continuada, para a grande maioria dos doentes incluídos, ultrapassava largamente o período mínimo de 1 ano. Assim, os resultados obtidos reflectem terapêuticas mantidas por períodos mais longos, embora não quantificados. Os resultados dos grandes ensaios clínicos mostram que o tempo requerido para uma efectiva redução do risco vascular é variável: o desenho dos estudos com estatinas ou com iecas/aras II engloba vários anos, mas as diferenças tornam-se significativas a partir do $1^{\circ}$ ano e, nalguns casos, em situações específicas de alto risco, após seis meses de tratamento continuado. Os antiagregantes plaquetários podem manifestar efectiva protecção mais precocemente-em situações de alto risco, como síndromes coronários agudos, as diferenças para o controlo podem revelarse em meses ou mesmo dias. ${ }^{33}$

Admite-se como teoricamente possível que os benefícios demonstrados para cada grupo terapêutico nos grandes ensaios clínicos possam ser potenciados por diferentes associações terapêuticas dos mesmos, em diferentes populações de risco cv e em diferentes estádios do processo aterosclerótico, a condição básica subjacente, que se pode evidenciar em múltiplas manifestações clínicas. ${ }^{30}$ Algumas destas associações terapêuticas foram já parcialmente estudadas, com resultados inconclusivos, quer no estudo SCAT («synergism of ACE inhibitors and cholesterol lowering therapy is possible, but unproven: no additional benefits were seen in the group receiving both drugs statins and ACE inhibitors in Scat study»), ${ }^{35}$ quer no estudo ALLHATt ( $« n o ~ b e-$ 


\begin{tabular}{|c|c|c|c|c|c|}
\hline \multirow{4}{*}{$\begin{array}{l}\text { Terapêutica } \\
\text { *Estatinas + Antiagreg }\end{array}$} & \multicolumn{2}{|c|}{ Controlos } & \multicolumn{2}{|c|}{ Casos } & \multirow{4}{*}{$\begin{array}{c}\text { Testes Estatísticos } \\
\text { Qui2: } 15,071 \quad P<0,001 \\
\text { OR: } 0,324(0,180-0,583)\end{array}$} \\
\hline & 183 & 72,6 & 131 & 89,1 & \\
\hline & 69 & 27,4 & 16 & 10,9 & \\
\hline & 252 & 100 & 147 & 100 & \\
\hline \multirow{3}{*}{ *lecas* + Antiagregantes } & 158 & 62,7 & 99 & 66,4 & \multirow{3}{*}{$\begin{array}{l}\text { Qui2: } 0,570 \quad P: 0,450 \\
\text { OR: } 0,849(0,555-1,299)\end{array}$} \\
\hline & 94 & 37,3 & 50 & 33,6 & \\
\hline & 252 & 100 & 149 & 100 & \\
\hline \multirow{3}{*}{ *lecas/Aras + Estatinas } & 182 & 71,9 & 122 & 83 & \multirow{3}{*}{$\begin{array}{l}\text { Qui2: } 6,231 \quad \text { P: } 0,013 \\
\text { OR: } 0,525(0,315-0,875)\end{array}$} \\
\hline & 71 & 28,1 & 25 & 17 & \\
\hline & 253 & 100 & 147 & 100 & \\
\hline \multirow{3}{*}{ *Antag Ca + Diuréticos } & 216 & 85,4 & 118 & 79,2 & \multirow{3}{*}{$\begin{array}{l}\text { Qui2: } 2,549 \text { P: } 0,110 \\
\text { OR: } 1,534(0,905-2,599)\end{array}$} \\
\hline & 37 & 14,6 & 31 & 20,8 & \\
\hline & 253 & 100 & 149 & 100 & \\
\hline \multirow{3}{*}{ *Estat+Antiag + iecas } & 198 & 78,3 & 136 & 91,3 & \multirow{3}{*}{$\begin{array}{l}\text { Qui2: } 11,301 \quad P: 0,001 \\
\text { OR: } 0,344(0,181-0,654)\end{array}$} \\
\hline & 55 & 21,7 & 13 & 8,7 & \\
\hline & 253 & 100 & 149 & 100 & \\
\hline \multirow{3}{*}{ *Antag ca+diur + iecas } & 225 & 88,9 & 126 & 84,6 & \multirow{3}{*}{$\begin{array}{l}\text { Qui2: } 1,177 \quad P: 0,278 \\
\text { OR: } 1,392(0,765-2,535)\end{array}$} \\
\hline & 28 & 11,1 & 23 & 15,4 & \\
\hline & 253 & 100 & 149 & 100 & \\
\hline \multirow{3}{*}{$\begin{array}{l}{ }^{* *} \text { Estatinas vs } \\
\text { Estatinas + Antiagregantes }\end{array}$} & 37 & 35,2 & 13 & 44,8 & \multirow{3}{*}{$\begin{array}{l}\text { Qui2: } 0,893 \quad P: 0,345 \\
\text { OR: } 0,670(0,291-1,542)\end{array}$} \\
\hline & 68 & 64,8 & 16 & 55,2 & \\
\hline & 105 & 100 & 29 & 100 & \\
\hline \multirow{3}{*}{$\begin{array}{l}\text { **Iecas/Aras II vs } \\
\text { lecas/Aras II + Antiagreg }\end{array}$} & 57 & 37,7 & 36 & 41,9 & \multirow{3}{*}{$\begin{array}{l}\text { Qui2: 0,389 P: 0,533 } \\
\text { OR: } 0,842(0,491-1,445)\end{array}$} \\
\hline & 94 & 62,3 & 50 & 58,1 & \\
\hline & 151 & 100 & 86 & 100 & \\
\hline \multirow{3}{*}{$\begin{array}{l}\text { **lecas/Aras II vs } \\
\text { lecas/Aras II + Estatinas }\end{array}$} & 80 & 53 & 60 & 71,4 & \multirow{3}{*}{$\begin{array}{l}\text { Qui2: } 7,628 \text { P: } 0,006 \\
\text { OR: } 0,451 \quad(0,255-0,798)\end{array}$} \\
\hline & 71 & 47 & 24 & 28,6 & \\
\hline & 151 & 100 & 84 & 100 & \\
\hline \multirow{3}{*}{$\begin{array}{l}\text { **Antag Ca vs } \\
\text { Antag Ca + Diuréticos }\end{array}$} & 26 & 41,3 & 16 & 34 & \multirow{3}{*}{$\begin{array}{l}\text { Qui2: } 0,596 \text { P: } 0,440 \\
\text { OR: } 1,361 \quad(0,621-2,984)\end{array}$} \\
\hline & 37 & 58,7 & 31 & 66 & \\
\hline & 63 & 100 & 47 & 100 & \\
\hline \multirow{3}{*}{$\begin{array}{l}{ }^{* *} \text { Antiagregantes } v s \\
\text { Antiagregantes + Estatinas }\end{array}$} & 56 & 45,2 & 47 & 74,6 & \multirow{3}{*}{$\begin{array}{l}\text { Qui2: } 14,636 \quad P<0,001 \\
\text { OR: } 0,280(0,144-0,547)\end{array}$} \\
\hline & 68 & 54,8 & 16 & 25,4 & \\
\hline & 124 & 100 & 63 & 100 & \\
\hline
\end{tabular}

Obs. *doentes com outra terapêutica vs determinada associação terapêutica; **doentes sob dada terapêutica vs associação terapêutica; OR: Odds Ratio. P: 0,05 Cl: $95 \%$

nefits in terms of all causes mortality or coronary and stroke events were apparent in patients on usual medication and on concomitant statins»). ${ }^{36} \mathrm{~A}$ análise exploratória, neste estudo, foi no sentido de pesquisar resultados favoráveis de determinadas associações terapêuticas comparativamente às demais terapêuticas, no geral. Não obstante os resultados positivos de algumas associações (dupla: estatinas e antiagregantes plaque- tários; tripla: estatinas, antiagregantes e iecas/aras II), pode apenas concluir-se, contudo, que as estatinas mantiveram a associação com a mortalidade independentemente de estarem ou não associadas àquelas terapêuticas (Quadro VII). Pesquisaram-se eventuais efeitos aditivos, comparando casos e controlos sujeitos a uma determinada terapêutica consoante faziam ou não outros fármacos: os doentes medicados com an- 
tiagregantes plaquetários, se sujeitos também a estatinas, parecem estar mais protegidos para o endpoint (Quadro VII). Este perfil verificou-se igualmente nos doentes sob iecas/aras II que faziam também estatinas. No entanto, a associação, quer de antiagregantes, quer de iecas/aras II, nos doentes que faziam estatinas, não revelou qualquer benefício adicional.

Deve referir-se que os resultados obtidos carecem de ponderação tendo em conta as limitações inerentes aos estudos de casos e controlos, bem como a determinados aspectos da metodologia. Primeiro, selecção de casos: para obviar à pouca fiabilidade dos registos de óbito na classificação da causa de morte, recorreu-se a entrevista semi-estruturada com o médico assistente (conjugando com dados do processo clínico), por forma a filtrar a fonte de dados disponível, o que foi considerado suficiente, dada a natureza exploratória deste trabalho (indivíduos cuja causa de morte foi reconhecidamente mal atribuída foram excluídos); segundo, identificação e selecção de controlos: o recurso à selecção de utilizadores regulares das consultas médicas aumentou a probabilidade de recolha de dados clínicos consistentes, mas este procedimento não foi extensivo aos casos; esta limitação é aceitável tendo em conta os dados recolhidos, quer sobre factores de risco (prevalentes nos casos), quer sobre as terapêuticas prescritas; terceiro, desenho do estudo: o tempo requerido de terapêutica continuada (critério de inclusão) de um ano, no mínimo, pode, à partida, ser insuficiente para discriminação de efeitos, tendo-se verificado, contudo, que a duração das terapêuticas foi, em média, bem superior; a variável «grupo terapêutico» foi definida com base no efeito de classe (três principais grupos), bem estabelecido para alguns medicamentos, menos bem para outros; não foram definidos critérios de avaliação/validação de dados relativos a aderência à medicação, equipotência das doses ou atingimento de metas laboratoriais ou outras preconizadas em guidelines; reconhecemos, contudo, a sua importância - o estudo REALITY, por exemplo, avaliou os padrões de prescrição das terapêuticas hipolipemiantes em locais representativos dos cuidados de saúde primários da Europa e concluiu que aproximadamente $60 \%$ dos europeus estudados não tinham alcançado os níveis de colesterol recomendados, $90 \%$ dos pacientes estavam com estatinas em regimes de média ou baixa equipotência; ${ }^{1}$ outro estudo recente, em doentes com fibrilhação auricular, chegou à conclusão que as terapêuticas antiarrítmicas utilizadas não estavam de acordo com as guidelines internacionais; ${ }^{40}$ admitimos que, no presente estudo, eventuais discrepâncias nessas variáveis poderão distribuir-se igualmente nos casos e nos controlos, e não deverão afectar a validade dos resultados.

\section{CONCLUSÕES}

As estatinas revelaram, neste estudo, associação significativa com menor mortalidade, no global e na maioria das sub-populações de risco analisadas, na linha dos resultados dos grandes ensaios clínicos. As duas outras classes terapêuticas estudadas, iecas/aras II e antiagregantes plaquetários, não obtiveram resultados com significado estatístico, embora o OR os coloque na faixa protectora.

Nos pressupostos deste estudo, os doentes sob estatinas parecem estar mais protegidos independentemente dos factores de risco (à excepção da doença coronária) e das terapêuticas concomitantes (redução estimada do risco de morte de causa vascular de $65 \%$ a $81 \%$ ).

Estes resultados foram observados em doentes com diferentes situações de risco, nomeadamente, eventos cv prévios, diabetes, «associação de 3 ou mais factores de risco», e não apenas nos doentes com dislipidémia. O estudo das associações terapêuticas contendo estatinas apenas revelou que estas mantiveram aparente efeito protector para o endpoint, isoladamente ou em associações duplas ou triplas (iecas/aras II e antiagregantes plaquetários). As estatinas revelaram benefícios adicionais nos doentes sob antiagregantes ou sob iecas/aras II.

O contributo relativo dos factores de risco para o endpoint, sublinha o impacto, à cabeça, do tabagismo e dos eventos cardiovasculares prévios.

Tratando-se dum estudo exploratório, estes resultados necessitam ser confirmados mediante investigações mais aprofundadas e em maior escala, preferencialmente a realizar em populações dos cuidados de saúde primários (estudos observacionais, por imperativo ético).

\section{REFERÊNCIAS BIBLIOGRÁFICAS}

1. Ganse EV, Laforest L, Alemão E, Davies G, Gutkin S, Yin D. Lipid-modifying therapy and attainment of cholesterol goals in Europe: the Return on Expenditure Achieved for Lipid Therapy (REALITY) study. Curr Med Res Opin 2005 Sep; 21 (9): 1389-99. 
2. Flather MD, Yusuf S, Køber L, Pfeffer M, Hall A, Murray G, et al. Long term ACE-inhibitor therapy in patients with heart failure or left ventricular dysfunction: a systematic overview of data from individual patients. Lancet 2000 May 6; 355 (9215): 1575-81.

3. SHEP Cooperative Research Group. Prevention of stroke by antihypertensive drug treatment in older persons with isolated systolic hypertension. Final results of the Systolic Hypertension in the Elderly Program (SHEP). JAMA 1991 Jun 26; 265 (24): 3255-64.

4. Staessen JAQ, Fagard R, Thijs L, Celis H, Arabidze GG,. Birkenhäger WH, et al. Randomised double-blind comparison of placebo and active treatment for older patients with isolated systolic hypertension. The Systolic Hypertension in Europe (SYST - EUR) Trial Investigators. Lancet 1997 Sep 13; 350 (9080): 757-64.

5. Dahlöf B, Devereux RB, Kjeldsen SE, Julius S, Beevers G, de Faire U, et al. Cardiovascular morbidity and mortality in the Losartan Intervention For Endpoint reduction in hypertension study (LIFE): a randomised trial against atenolol. Lancet 2002 Mar 23; 359 (9311): 995-1003.

6. The SOLVD investigators. Effect of enalapril on mortality and the development of heart failure in asymptomatic patients with reduced left ventricular ejection fractions. N Engl J Med 1992 Sep 3; 327 (10): 685-91.

7. Pfeffer MA, Braunwald E, Moyé LA, Basta L, Brown EJ Jr, Cuddy TE, et al. Effect of captopril on mortality and morbidity in patients with left ventricular dysfunction after myocardial infarction: results of the survival and ventricular enlargement trial (SAVE). N Engl J Med 1992 Sep 3; 327 (10): 669-77.

8. The Acute Infarction Ramipril Efficacy (AIRE) study investigators. Effect of ramipril on mortality and morbidity of survivors of acute myocardial infarction with clinical evidence of heart failure. Lancet 1993 Oct 2; 342 (8875): 821-8.

9. Køber L, Torp-Pedersen C, Carlsen JE, Bagger H, Eliasen P, Lyngborg K, et al. A clinical trial of the angiotensin-converting-enzyme inhibitor trandolapril in patients with left ventricular dysfunction after myocardial infarction (TRACE). N Engl J Med 1995 Dec 21; 333 (25): 1670-6.

10. The Heart Outcomes Prevention Evaluation (HOPE) study investigators. Effects of an angiotensin-converting-enzyme inhibitor, ramipril, on cardiovascular events in high-risk patients. N Engl J Med 2000 Jan 20; 342 (3): 145-53.

11. Maggioni AP, Anand I, Gottlieb SO, Latini R, Tognoni G, Cohn JN. Effect of valsartan on morbidity and mortality in patients with heart failure not receiving angiotensin-converting enzyme inhibitors (VAL-HEFT). J Am Coll Cardiol 2002 Oct 16; 40 (8): 1414-21.

12. Pfeffer M, Swedberg K, Granger C, et al: Effects of candesartan on mortality and morbidity in patients with chronic heart failure - the CHARM-Overall programme; Lancet 2003; 362: 759-66.

13. Fox KM, EURopean trail On reduction of cardiac events with Perindopril in stable coronary Artery disease Investigators. Efficacy of perindopril in reduction of cardiovascular events among patients with stable coronary artery disease: randomised, double-blind, placebo-controlled, multicentre trial (the EUROPA study). Lancet 2003 Sep 6; 362 (9386): 782-8.

14. Sever PS, Dahlöf B, Poulter NR, Wedel H, Beevers G, Caulfield M, et al. Prevention of coronary and stroke events with atorvastatin in hypertensive patients who have average or lower-than-average cholesterol concentrations, in the Anglo-Scandinavian Cardiac Outcomes Trial Lipid Lowering Arm (ASCOT-LLA): a multicentre randomised controlled trial. Lancet 2003 Apr 5; 361 (9364): 1149-58.
15. Scandinavian Simvastatin Survival Study (4S) Group. Randomized trial of cholesterol lowering in 4444 patients with coronary heart disease: the Scandinavian Simvastatin Survival Study (4S). Lancet 1994 Nov 19; 344 (8934): 1383-9.

16. Shepherd J, Cobbe SM, Ford I, Isles CG, Lorimer AR, MacFarlane PW, et al. Prevention of coronary heart disease with pravastatin in men with hypercholesterolemia. N Engl J Med 1995 Nov 16; 333 (20): 1301-7.

17. Sacks FM, Pfeffer MA, Moye LA, Rouleau JL, Rutherford JD, Cole TG, et al. The effect of pravastatin on coronary events after myocardial infarction in patients with average cholesterol levels. N Engl J Med 1996 Oct 3; 335 (14): 1001-9.

18. Serruys PW, de Feyter P, Macaya C, Kokott N, Puel J, Vrolix M, et al. Fluvastatin for prevention of cardiac events following successful first percutaneous coronary intervention: a randomizes controleed trial. JAMA 2002 Jun 26; 287 (24): 3215-22.

19. Shepherd J, Blauw GJ, Murphy MB, Bollen EL, Buckley BM, Cobbe SM, et al. Pravastatin in elderly individuals at risk of vascular disease (PROSPER): a randomised controlled trial. Lancet 2002 Nov 23; 360 (9346): 1623-30.

20. Heart Protection Study (HPS) Collaborative Group. MRC/BHF heart protection study of cholesterol lowering with simvastatin in 20,536 highrisk individuals: a randomised placebo-controlled trial. Lancet 2002 Jul 6; 360 (9326): 7-22.

21. Pedersen TR, Faergemen O, Kastelein JJ, Olsson AG, Tikkanen MJ, Holme I, et al. High-dose atorvastatin vs usual-dose simvastatin for secondary prevention after myocardial infarction: the IDEAL study: a randomized controlled trial. JAMA 2005 nov 16; 294 (19): 2437-45.

22. Downs JR, Clearfield M, Weis S, Whitney E, Shapiro DR, Beere PA, et al. Primary prevention of acute coronary events with lovastatin in men and women with average cholesterol levels: results of AFCAPS/ TexCAPS. JAMA 1998 May 27; 279 (20: 1615-22.

23. The Long-Term Intervention with Pravastatin in Ischaemic Disease (LIPID) Study Group. Prevention of cardiovascular events and death with pravastatin in patients with coronary heart disease and a broad range of initial cholesterol levels. N Engl J Med 1998 Nov 5; 339 (19): 1349-57.

24. Schwartz GG, Olsson AG, Ezekowitz MD, Ganz P, Oliver MF, Waters D, et al. Effects of atorvastatin on early recurrent ischemic events in acute coronary syndromes: the MIRACL study: a randomized controlled trial. JAMA 2001 Apr 4; 285 (13): 1711-8.

25. Antiplatelet Trialists' Colaboration. Secondary prevention of vascular disease by prolonged antiplatelet treatment. BMJ 1988 Jan 30; 296 (6618): 320-31.

26. Antiplatelet Trialists' Colaboration. Collaborative overview of randomized trials of antiplatelet therapy: I: Prevention of death, myocardial infarction, and stroke by prolonged antiplatelet therapy in various categories of patients. BMJ 1994 Jan 8; 308 (6921): 81-106.

27. Yusuf S, Wittes J, Friedman L. Overview of results of randomized clinical trials in heart disease. II: Unstable angina, heart failure, primary prevention with aspirin, and risk factor modification. JAMA 1988 Oct 21; 260 (15): 2259-63.

28. Eidelman RS, Hebert PR, Weisman SM, Hennekens $\mathrm{CH}$. An update on aspirin in the primary prevention of cardiovascular disease. Arch Intern Med 2003 Sep 22; 163 (17): 2006-10.

29. Atrial Fibrillation (AFI) Investigators. Risk factors for stroke and efficacy of antithrombotic therapy in atrial fibrillation: analysis of pooled data from five randomized controlled trials. Arch Intern Med 1994 Jul 11; 154 (13): 1449-57. 
30. CAPRIE Steering Committee. A randomized, blinded, trial of clopidogrel versus aspirin in patients at risk of ischemic events (CAPRIE). Lancet 1996 Nov 16; 348 (9038): 1329-39.

31. ISIS-2 (Second International Study of Infarct Survival) Collaborative Group. Randomised trial of intravenous streptokinase, oral aspirin, both, or neither among 17,187 cases of suspected acute myocardial infarction (ISIS-2). Lancet 1988 Aug 13; 2 (8607): 349-60.

32. Ridker PM, Cook NR, Lee IM, Gordon D, Gaziano JM, Manson JE, et al. A randomized trial of low-dose aspirin in the primary prevention of cardiovascular disease in women. N Engl J Med 2005 Mar 31; 352 (13): 1293-304.

33. Yusuf S, Zhao F, Mehta SR, Chrolavicius S, Tognoni G, Fox KK; Clopidogrel in Unstable Angina to Prevent Recurrent Events Trial Investigators. Effects of clopidogrel in addition to aspirin in patients with acute coronary syndromes without ST-segment elevation. N Engl J Med 2001 Aug 16; 345 (7): 404-502.

34. Stroke Prevention in Atrial Fibrillation (SPAF) investigators: risk factors for thromboembolism during aspirin therapy in patients with atrial fibrillation: the stroke prevention in atrial fibrillation. J Stroke Cerebrovasc Dis 1955; 5: 147-57.

35. Teo KK, Burton JR, Buller CE, Plante S, Catellier D, TymchakW, et al. Longterm effects of cholesterol lowering and angiotensin-converting enzyme inhibition on coronary atherosclerosis: the Simvastatin/Enalapril Coronary Atherosclerosis Trial (SCAT). Circulation 2000 Oct 10; 102 (15): 1748-54.

36. ALLHAT Officers and Coordinators for the ALLHAT Collaborative Research Group. Major outcomes in high-risk hypertensive patients randomized to angiotensin-converting enzyme inhibitor or calcium channel blocker vs diuretic: the Antihypertensive and Lipid-Lowering Treatment to Prevent Heart Attack Trial (ALLHAT). JAMA 2002 Dec 18; 288 (23): 2981-97.

37. Gaede $P$, Vedel $P$, Larsen $N$, Jensen $G V$, Parving $H H$, et al. Multifactor intervention and cardiovascular disease in patients with type 2 diabetes. N Engl J Med 2003 Jan 30; 348 (5): 383-93.

38. Grupo de Trabalho do Prontuário (INFARMED). Prontuário Terapêutico. $5^{\text {a }}$ ed. Lisboa: INFARMED / Ministério da Saúde; 2004.

39. OMS. Oitava Classificação Internacional de Doenças (CID 8). Geneva: OMS; 1965

40. Ascenção P. Fibrilhação auricular e prevenção do tromboembolismo: estudo numa população de utentes de centros de saúde. Rev Port Clin Geral 2006 Jan-Fev; 22 (1): 13-24.

\section{AGRADECIMENTOS}

A todos os médicos que com a sua colaboração viabilizaram a realização deste trabalho.

Ao Mestre Pedro Aguiar, consultor estatístico, pela sua orientação no tratamento de dados e revisão metodológica.

\section{ENDEREÇO PARA CORRESPONDÊNCIA}

Idalmiro Rocha Carraça

Rua José D’Esaguy $n^{\circ} 14,1^{\circ}$ esquerdo, 1700 Lisboa

Tlm: 966554907

E-mail: idalcarraca@gmail.com

Recebido em 02/12/2008

Aceite para publicação em 13/03/2009

\begin{abstract}
Background: Preventive therapy of cardiovascular (cv) events is changing clinical practice, providing their effectiveness, according to the results of international randomized trials. The new antihypertensive agents, statins, and antiplatelet treatment account for significant relative risk reduction.

Objectives: To evaluate, comparatively and retrospectively, cv death risk reduction associated to those medications, in patients submitted to conventional primary health care; to evaluate therapeutic associations and additional benefits of concomitant medications; to explore risk factors' contribution to vascular death.

Design: Cases and controls study, based on usual patterns of medication for cv disease (statins, antihypertensive agents or antiplatelet treatment), was designed to evaluate $c v$ death risk reduction in a minimum of a one-year period of fully prescription. A total of 402 patients were assigned and distributed as cases and controls: cases were identified from health centre obit data base, including all individuals whose death occurred in the years 2003 and 2004, registered as related with vascular cause; controls were randomized after selection of patients with successive appointments to their family doctors in the last few years; homogeneity as for sex and age was granted.

Statistics methods: Qui square for testing homogeneity by sex, age and risk factors distribution; Odds Ratio (OR) for risk estimate of death from vascular cause by therapeutic group and by risk factors; Logistic regression (OR adjusted for risk factors). $95 \%$ confidence interval.

Results: Statins shown in this study to be associated to cv death risk (relative risk reduction estimate: 65 to $81 \%$ ).

Conclusions: The results suggest that, in the patients involved, statins have contributed to important and significant cv death risk reduction; «smoke» and previous cv events were the prominent risk factors.
\end{abstract}

Keywords: Risk Reduction; Preventive Therapy. 